\title{
FROM BOSE CONDENSATION TO QUANTUM GRAVITY AND BACK
}

\author{
K. N. Ilinski ${ }^{1,2}$, A. S. Stepanenko ${ }^{1,3}$ \\ ${ }^{1}$ School of Physics and Space Research, University of Birmingham, Birmingham B15 2TT, United Kingdom \\ ${ }^{2}$ Institute of Spectroscopy, Russian Academy of Sciences, Troitsk, Moscow region, 142092, Russian Federation \\ ${ }^{3}$ Theoretical Department, St-Petersburg Nuclear Physics Institute, Gatchina, St-Petersburg, 188350, Russian Federation
}

(Received March 8, 1998)

\begin{abstract}
We account for the interaction of the Bose-condensed fraction with the normal phase in an effective dynamical equation such as the Gross-Pitaevskii equation. We show that the low-energy excitations can be treated as sound waves with speed dependent on the condensate density. This allows us to reduce the problem to the calculation of the determinant of the Laplace operator on a curved space and apply standard methods of quantum gravity to get the leading logarithmic contribution of the determinant. This produces the first quantum correction due to the noncondensed fraction to the Gross-Pitaevskii equation for the condensate. The correction describes an additional quantum pressure in the condensate and evaporation-condensation effects.
\end{abstract}

Key words: Bose-condensation, effective action, quantum gravity.

PACS number(s): 05.30.Jp, 04.65.+v, 02.40.-k

\section{I.}

In the last few years there has been a new growth of the interest in Bose-Einstein condensation (in particular, for spatially inhomogeneous situations) due to the recent success of the experimental observation of BoseEinstein condensation for systems of spin polarized magnetically trapped atomic gases at ultra-low temperatures [1-3] and further investigation of their collective properties [4]. These experimental results stimulated the development of a theory of a nonuniform Bose condensate and its collective excitations. Moreover, new exciting problems such as a description of the evolution of the Bose condensate from a relaxed trap, stability and dynamics of the collapse of the condensate for $\mathrm{Li}^{7}$ atoms, collective excitations, heating-cooling phenomena, various coherence effects for the condensate and so on are being posed both theoretically and experimentally.

All the problems listed above require an accurate account of the interaction of the condensate with the noncondensed fraction. Formally, the problem can be tackled using the Bogoliubov-Popov equations which represent a set of coupled linear and nonlinear Schrödinger equations; the nonlinear Schrödinger equation (for the condensate fraction) coupled via a potential term with a pair of Schrödinger equations (for the excited modes) whose potentials, on their own, depend on the solution of the nonlinear Schrödinger equation. To find an effective equation for the condensate we need to resolve a pair of Schrödinger equations with arbitrary potentials and find the solutions of the equations as functionals of the potentials. It is obvious that this is hardly a realistic task to perform.

However, as we show in the paper, it is possible to calculate the corrections from the non-condensed fraction and, surprisingly, they look very simple in specially chosen notations. The answer comes from exploiting a technique originally developed to solve problems in quan- tum gravity, which seems to be quite far from the Bose condensation. To make use of it, we note that the lowenergy modes of the non-condensed fraction can be considered as sound waves whose velocity depends on the density and velocity of the condensate. This shows that the interaction of the modes with the condensate has the same nature as the interaction of matter fields with the background gravity field. In this framework the GrossPitaevskii equation is an analogue of the Einstein equation where the energy-momentum tensor is due to presence of quantum matter fields, that is excited modes. It is not surprising then that to calculate the correction we use methods from quantum gravity. More precisely, we use functional integration, $\zeta$ - function renormalization for determinants of elliptic operators and the Seeley expansion of the heat-kernel for the Laplace operator on a curved space in an external field. The final form of the correction to the free energy of the condensate, written in terms of the curvature tensor then has a very simple and manageable form (6).

\section{II.}

We are looking for the effective action which describes all physical quantities in the system (for an easy introduction to the formalism of the effective action see, for example, Appendix A of Ref. [5]). For example, the effective action provides all of the Green's functions in the same approximation used to calculate the effective action itself. The equations of motion for the average values of field operators are given by the equations for a minimum of the effective action. We obtain them in a hydrodynamic one-loop approximation. To clarify the description the hydrodynamical variables, density and velocity, should be used instead of microscopic variables. More precisely, we start with the action 


$$
\frac{S}{\hbar}=\int_{-\infty}^{\infty} \mathrm{d} \tau \int \mathrm{d} V\left\{i \psi^{+} \frac{\partial \psi}{\partial \tau}-\left[\nabla \psi^{+} \nabla \psi+(v-\mu) \psi^{+} \psi+l\left(\psi^{+} \psi\right)^{2}\right]\right\}
$$

and change field variables to the hydrodynamic ones:

$$
\psi(x, \tau)=\sqrt{\rho(x, \tau)} e^{-i \varphi(x, \tau)}, \quad \psi^{+}(x, \tau)=\sqrt{\rho(x, \tau)} e^{i \varphi(x, \tau)} .
$$

This transforms the action (1) into the form (up to a complete derivative term):

$$
S=\int_{-\infty}^{\infty} \mathrm{d} \tau \int \mathrm{d} V\left\{\frac{\partial \varphi}{\partial \tau} \rho-(\nabla \sqrt{\rho})^{2}-\rho(\nabla \varphi)^{2}-(v-\mu) \rho-l \rho^{2}\right\}
$$

It is not difficult to see that the classical equations of motion for the action lead to the equations:

$$
\begin{gathered}
\frac{\partial \mathbf{c}}{\partial \tau}+\nabla\left(\frac{\mathbf{c}^{2}}{2}+2 v-2 \mu+4 l \rho-\frac{2}{\sqrt{\rho}} \nabla^{2} \sqrt{\rho}\right)=0, \\
\frac{\partial \rho}{\partial \tau}+\nabla(\mathbf{c} \cdot \rho)=0,
\end{gathered}
$$

which are equivalent to the Gross-Pitaevskii equation [6] after the introduction of the velocity variable $\mathbf{c}=-2 \nabla \varphi$ instead of $\varphi$. These equations look like hydrodynamic equations for an irrotational compressible fluid which is the condensate superfluid.

Now let us shift our variables in (1) by the zero-order mean field solution (the condensate amplitude) to find the one loop quantum correction for the effective action:

$$
\psi \rightarrow \psi+\chi, \quad \psi^{+} \rightarrow \psi^{+}+\chi^{+}
$$

and keep only quadratic terms of $\chi$ and $\chi^{+}$since it can be shown [5] that this is the only relevant contribution for the case of trapped gases. Then the action (1) transforms to $S\left(\psi, \psi^{+}\right)+S_{1}\left(\psi, \psi^{+}, \chi, \chi^{+}\right)$where the term $S_{1}$ can be written as:

$$
S_{1}\left(\psi, \psi^{+}, \chi, \chi^{+}\right)=\int_{-\infty}^{\infty} \mathrm{d} \tau \int \mathrm{d} V\left\{i \chi^{+} \frac{\partial \chi}{\partial \tau}-\left[\nabla \chi^{+} \nabla \chi+\left\{\dot{\varphi}-(\nabla \varphi)^{2}+2 l \rho\right\} \chi^{+} \chi-l e^{2 i \varphi} \rho \chi^{+} \chi^{+}-l e^{-2 i \varphi} \rho \chi \chi\right]\right\} .
$$

In the last equation the potential $v$ was excluded using the mean field equation of motion neglecting the kinetic term. The Hamiltonian corresponding to $S_{1}$ is

$$
H=\int \mathrm{d} V\left[\nabla \hat{\chi}^{+} \nabla \hat{\chi}+\left\{\dot{\varphi}-(\nabla \varphi)^{2}+2 l \rho\right\} \hat{\chi}^{+} \hat{\chi}-l e^{2 i \varphi} \rho \hat{\chi}^{+} \hat{\chi}^{+}-l e^{-2 i \varphi} \rho \hat{\chi} \hat{\chi}\right]
$$

Now we perform the following canonical transformation:

$$
\hat{\chi}=\sqrt{\rho} \mathrm{e}^{i \varphi}\left[\frac{\hat{\sigma}}{2 \rho}+i \hat{\alpha}\right], \quad \hat{\chi}^{+}=\sqrt{\rho} \mathrm{e}^{-i \varphi}\left[\frac{\hat{\sigma}}{2 \rho}-i \hat{\alpha}\right] .
$$

The Hamiltonian takes the form

$$
\begin{aligned}
& H=\int \mathrm{d} V\left[\rho(\nabla \hat{\alpha})^{2}+\rho \dot{\varphi} \hat{\alpha}^{2}+\left\{l+\frac{\dot{\varphi}}{4 \rho}\right\} \hat{\sigma}^{2}+\hat{\sigma}(\nabla \varphi \nabla \hat{\alpha})-\hat{\alpha}(\nabla \varphi \nabla \hat{\sigma})+\frac{1}{2} \Delta \varphi \hat{\sigma} \hat{\alpha}\right. \\
& \left.-\frac{1}{4 \rho^{2}} \hat{\sigma} \nabla \rho \nabla \hat{\sigma}+\frac{(\nabla \rho)^{2}}{4 \rho} \hat{\alpha}^{2}+\frac{1}{4 \rho}(\nabla \hat{\sigma})^{2}+\frac{\nabla \rho \nabla \varphi}{\rho} \hat{\alpha} \hat{\sigma}+\frac{(\nabla \rho)^{2}}{16 \rho^{3}} \hat{\sigma}^{2}+\hat{\alpha} \nabla \rho \nabla \hat{\alpha}\right] .
\end{aligned}
$$


Last eight terms vanish in the hydrodynamical limit since $\rho$ is a large variable $(\nabla \varphi \sim \sqrt{l \rho}$ and $\dot{\varphi} \sim l \rho)$, so we have

$$
H=\int \mathrm{d} V\left[\rho(\nabla \hat{\alpha})^{2}+\rho \dot{\varphi} \hat{\alpha}^{2}+\left\{l+\frac{\dot{\varphi}}{4 \rho}\right\} \hat{\sigma}^{2}+\hat{\sigma}(\nabla \varphi \nabla \hat{\alpha})-\hat{\alpha}(\nabla \varphi \nabla \hat{\sigma})\right] .
$$

The corresponding action (in the Feynman functional integral) is

$$
S_{1}=\int_{-\infty}^{\infty} \mathrm{d} \tau \int \mathrm{d} V\left\{\frac{\partial \alpha}{\partial \tau} \sigma-\left[\rho(\nabla \alpha)^{2}+\rho \dot{\varphi} \alpha^{2}+\left\{l+\frac{\dot{\varphi}}{4 \rho}\right\} \sigma^{2}+2 \sigma(\nabla \varphi \nabla \alpha)\right]\right\}
$$

Integrating the functional integral over the field $\sigma$ we get the action for $\alpha$ field only:

$$
S_{1}=\int_{-\infty}^{\infty} \mathrm{d} \tau \int \mathrm{d} V\left\{-\rho(\nabla \alpha)^{2}+f\left(\frac{\partial \alpha}{\partial \tau}-2 \nabla \varphi \nabla \alpha\right)^{2}-\rho \dot{\varphi} \alpha^{2}\right\}
$$

where $f=\frac{1}{4 l+\dot{\varphi} / \rho}$. The corresponding determinant from the integration does not contribute to the effective action at this order of the perturbation theory.

Now we will once again use the existence of the large parameter $\rho$. Let us introduce the following large number $\rho_{0} \equiv \max \{\rho\}$ such that $\tilde{\rho}=\frac{\rho}{\rho_{0}} \sim 1$, and new space-time variables $t \equiv 4 l \rho_{0} \tau$ and $y_{i} \equiv \sqrt{4 l \rho_{0}} x_{i}$. The action $S_{1}$ can be represented as

$$
\begin{aligned}
S_{1} & =\frac{1}{\sqrt{64 \rho_{0} l^{3}}} \int_{-\infty}^{\infty} \mathrm{d} t \int \mathrm{d} \tilde{V}\left\{-\tilde{\rho}(\tilde{\nabla} \alpha)^{2}+\tilde{f}\left(\frac{\partial \alpha}{\partial t}-\tilde{\mathbf{v}} \tilde{\nabla} \alpha\right)^{2}-\tilde{\rho} \dot{\varphi} \alpha^{2}\right\} \\
& \equiv-\frac{1}{\sqrt{64 \rho_{0} l^{3}}} \int_{-\infty}^{\infty} \mathrm{d} t \int \mathrm{d} \tilde{V}\left[A^{\mu \nu} \partial_{\mu} \alpha \partial_{\nu} \alpha+\tilde{\rho} \dot{\varphi} \alpha^{2}\right]
\end{aligned}
$$

where

$$
\tilde{\mathbf{v}}=2 \tilde{\nabla} \varphi, \quad \tilde{f}=4 l f=\frac{1}{1+\dot{\varphi} / \tilde{\rho}}
$$

and the matrix $A$ has the form

$$
A=\left(\begin{array}{cccc}
\tilde{f} & \tilde{f} \tilde{v}_{1} & \tilde{f} \tilde{v}_{2} & \tilde{f} \tilde{v}_{3} \\
\tilde{f} \tilde{v}_{1} & \tilde{\rho}-\tilde{f} \tilde{v}_{1}^{2} & -\tilde{f} \tilde{v}_{1} \tilde{v}_{2} & -\tilde{f} \tilde{v}_{1} \tilde{v}_{3} \\
\tilde{f} \tilde{v}_{2} & -\tilde{f} \tilde{v}_{1} \tilde{v}_{2} & \tilde{\rho}-\tilde{f} \tilde{v}_{2}^{2} & -\tilde{f} \tilde{v}_{2} \tilde{v} \tilde{v}_{3} \\
\tilde{f} \tilde{v}_{3} & -\tilde{f} \tilde{v}_{1} \tilde{v}_{3} & -\tilde{f} \tilde{v}_{2} \tilde{v}_{3} & \tilde{\rho}-\tilde{f} \tilde{v}_{3}^{2}
\end{array}\right)
$$

Our next step is to cast the action in the covariant form. To do this we introduce auxiliary metric $\tilde{g}_{\mu \nu}$ such that

$$
A^{\mu \nu}=\frac{\tilde{g}^{\mu \nu}}{\sqrt{-\operatorname{det}\left(\left\|\tilde{g}^{\mu \nu}\right\|\right)}}, \quad \tilde{g}^{\mu \nu}=\frac{A^{\mu \nu}}{\sqrt{-\operatorname{det}\left(\left\|A^{\mu \nu}\right\|\right)}}
$$

This leads to the form for the metric $\left\|\tilde{g}_{\mu \nu}\right\|$ :

$$
\left\|\tilde{g}_{\mu \nu}\right\|=\tilde{\rho}^{1 / 2} \tilde{f}^{1 / 2}\left(\begin{array}{cccc}
-\tilde{\rho} / \tilde{f}+\tilde{v}^{2} & \tilde{v}_{1} & \tilde{v}_{2} & \tilde{v}_{3} \\
\tilde{v}_{1} & 1 & 0 & 0 \\
\tilde{v}_{2} & 0 & 1 & 0 \\
\tilde{v}_{3} & 0 & 0 & 1
\end{array}\right)
$$

In this metric the action has a covariant form

$$
S_{1}=-\frac{1}{\sqrt{64 \rho_{0} l^{3}}} \int \mathrm{d} y \sqrt{-\tilde{g}}\left[\alpha \tilde{\Delta} \alpha+\tilde{E} \alpha^{2}\right]
$$


where

$$
\tilde{E}=\frac{\tilde{\rho} \dot{\varphi}}{\sqrt{-\tilde{g}}}=\frac{\dot{\varphi}}{\sqrt{\tilde{\rho} \tilde{f}}}, \quad \tilde{\Delta}=-\frac{1}{\sqrt{-\tilde{g}}} \partial_{\mu} \tilde{g}^{\mu \nu} \sqrt{-\tilde{g}} \partial_{\nu} .
$$

Now the effective action is $\Gamma_{1}=-\frac{1}{2} \operatorname{tr} \ln \left[\left(64 \rho_{0} l^{3}\right)^{-1 / 2}(\tilde{\Delta}+\tilde{E})\right]$ with Laplace operator $\tilde{\Delta}=-\frac{1}{\sqrt{-\tilde{g}}} \partial_{\mu} \tilde{g}^{\mu \nu} \sqrt{-\tilde{g}} \partial_{\nu}$.

At this point the quantum gravity analogy comes into the game and let us evaluate $\Gamma_{1}$ using $\zeta$-function regularization [7] for $\frac{1}{2} \operatorname{tr} \ln \left[\left(64 \rho_{0} l^{3}\right)^{-1 / 2}(\tilde{\Delta}+\tilde{E})\right]$ after performing the Wick rotation to make the operator elliptic. In this regularization the quantity is finite and has the following property:

$$
\operatorname{tr} \ln \left[\left(64 \rho_{0} l^{3}\right)^{-1 / 2}(\tilde{\Delta}+\tilde{E})\right]=\operatorname{tr} \ln [\tilde{\Delta}+\tilde{E}]-\frac{1}{2} \operatorname{tr} \ln \left(64 \rho_{0} l^{3}\right)\left(\Phi_{0}(\tilde{\Delta}+\tilde{E})-L(\tilde{\Delta}+\tilde{E})\right),
$$

where $\Phi_{0}(\tilde{\Delta}+\tilde{E}) \equiv \int \mathrm{d} y \mathrm{~d} \tau \sqrt{-\tilde{g}} \Psi_{0}(\tilde{\Delta}+\tilde{E})$ is the so-called zeroth Seeley coefficient and $L$ is a number of zero modes. For our goal is important to realize that in the regularization $\operatorname{tr} \ln [\tilde{\Delta}+\tilde{E}]$ is of order of the zeroth Seeley coefficient which, on its own, is of order unity.

The existence of the large parameter in the system allows us to make the next step. Indeed, the multiplier $\left(64 \rho_{0} l^{3}\right)^{-1 / 2}$ gives us the possibility to express the main contributions to the determinant such as

$$
\operatorname{tr} \ln \left[\left(64 \rho_{0} l^{3}\right)^{-1 / 2}(\tilde{\Delta}+\tilde{E})\right] \sim-\frac{1}{2} \operatorname{tr} \ln \left(64 \rho_{0} l^{3}\right) \int d x d t\left(\Psi_{0}(\tilde{\Delta}+\tilde{E})-L(\tilde{\Delta}+\tilde{E})\right)
$$

The Seeley coefficient $\Psi_{0}$ for the Laplace operator in $4 \mathrm{D}$ is well-known and can be found, for example, in Ref. [7]:

$$
\begin{gathered}
\Psi_{0}=\frac{\sqrt{-g}}{(4 \pi)^{2}}\left(-\frac{1}{30} \nabla^{2} R+\frac{1}{72} R^{2}-\frac{1}{180} R_{\mu \nu} R^{\mu \nu}+\frac{1}{180} R_{\mu \nu \sigma \rho} R^{\mu \nu \sigma \rho}\right. \\
\left.+\frac{1}{6} E R+\frac{1}{2} E^{2}-\frac{1}{6} \nabla^{2} E\right) .
\end{gathered}
$$

where $R, R_{\mu \nu}$ and $R_{\mu \nu \sigma \rho}$ are the scalar curvature, Ricci and Riemann tensors correspondingly [8].

In all physical applications the number of zero-modes does not change and we can drop the term with $L(\tilde{\Delta}+\tilde{E})$. Returning to the initial variables and performing inverse Wick rotation, all curvature tensors and the metric are written in the "physical" variables (i.e. without tildes). Moreover $\ln \left(64 \rho_{0} l^{3}\right) \gg \ln (\tilde{\rho})$ so that we can substitute $\ln \left(64 \rho l^{3}\right)$ instead of $\ln \left(64 \rho_{0} l^{3}\right)$. Summarizing, we obtain an expression for the first quantum correction to the effective action:

$$
\Gamma_{1}=\frac{1}{4} \int \mathrm{d} x \mathrm{~d} \tau \sqrt{-g} \ln \left(64 \rho l^{3}\right) \Psi_{0}(\Delta+E)
$$

with the metric

$$
\left\|g_{\mu \nu}\right\|=\rho^{1 / 2} f^{1 / 2}\left(\begin{array}{cccc}
-\rho / f+v^{2} & v_{1} & v_{2} & v_{3} \\
v_{1} & 1 & 0 & 0 \\
v_{2} & 0 & 1 & 0 \\
v_{3} & 0 & 0 & 1
\end{array}\right)
$$

and

$$
v_{i} \equiv 2 \partial_{i} \varphi, \quad f=\frac{1}{4 l+\dot{\varphi} / \rho}, \quad E=\frac{\dot{\varphi}}{\sqrt{\rho f}} .
$$

Equation (6) is the central equation of the paper.

Miminizing the effective action (2) with the correction (6), the equations of motion for the condensate can be derived as:

$$
\frac{\partial \varphi}{\partial \tau}-(\nabla \varphi)^{2}-v+\mu-2 l \rho+\frac{1}{4} \ln \left(64 \rho l^{3}\right)\left[\frac{\partial g_{\mu \nu}}{\partial \rho} \frac{\delta \Psi_{0}}{\delta g_{\mu \nu}}+\frac{\partial E}{\partial \rho} \frac{\delta \Psi_{0}}{\delta E}\right]=0
$$




$$
-\frac{\partial \rho}{\partial \tau}+2 \nabla(\nabla \varphi \cdot \rho)+\frac{1}{4} \ln \left(64 \rho l^{3}\right)\left[\int \mathrm{d}^{4} y \frac{\delta g_{\mu \nu}(x)}{\delta \varphi(y)} \frac{\delta \Psi_{0}}{\delta g_{\mu \nu}(x)}-\frac{\partial}{\partial \tau}\left(\frac{\partial E}{\partial \dot{\varphi}} \frac{\delta \Psi_{0}}{\delta E}\right)\right]=0 .
$$

The equations substitute for the Gross-Pitaevskii equation and generalize Eqns $(3,4)$ to account for the interaction of the Bose-condensate with the non-condensed fraction in the one-loop approximation.

\section{III.}

In conclusion, we have calculated the one-loop quantum correction for the effective action of the condensate. The correction comes from the interaction of the condensate with low-energy excited modes. To this end, we considered the excited modes as quantum particles moving in curved space whose metric (gravity field) was defined by the density and the velocity of the condensate. The equations of motion for the condensate are then analo- gous to the equations for the gravitational field where the right hand side (the energy-momentum tensor) is due to the presence of matter fields. The analysis does not depend on details of the external potential and can be used for a variety of problems. The only simplification is the common hydrodynamic approximation.

\section{ACKNOWLEDGMENT}

We are grateful to Keith Burnett and Mike Gunn for the discussions of the problem. This work was supported by the Grant of Russian Fund of Fundamental Investigations N 95-01-00548 and by the UK EPSRC Grants GR/L29156, GR/K68356.
[1] M. H. Anderson, J. R. Ensher, M. R. Matthews, C. E. Wieman, and E. A. Cornell, Science 269, 198 (1995).

[2] C. C. Bradley, C. A. Sackett, J. J. Tollett, and R. G. Hulet, Phys. Rev. Lett. 75, 1687 (1995).

[3] K. B. Davis, M.-O. Mewes, M. R. Andrews, N. J. van Druten, D. S. Durfee, D. M. Kurn, and W. Ketterle, Phys. Rev. Lett. 75, 3969 (1995).

[4] D. S. Jin, J. R. Ensher, M. R. Matthews, C. E. Wieman and E. A. Cornell, Phys. Rev. Lett. 77, 420 (1996).

[5] K. N. Ilinski and A. S. Stepanenko, preprint cond- mat/9607202 (1996).

[6] S. Stringari, Phys. Rev. Lett. 77, 2360 (1996).

[7] Albert S. Schwarz: Quantum Field Theory and Topology, Springer-Verlag, 1993.

[8] B. A. Dubrovin, A. T. Fomenko, S. P. Novikov, Modern Geometry - Methods and Applications (Springer-Verlag, 1984); S. Weinberg, Gravitation and cosmology: principles and applications of the general theory of relativity (John Wiley \& Sons, Inc. New York London Sydney Toronto, 1972). 